\title{
Differential Effects of Amyloid-Beta Treatment on both Mitochondrial Function and Calcium Homeostasis at Nerve Terminals and Glia
}

\author{
D. R. Giovannucci and C. A. Brink \\ Department of Neurosciences, Medical University of Ohio, 3035 Arlington Ave, Toledo OH 43614
}

There is strong evidence that in Alzheimer's Disease (AD), synapses are impaired before neurons degenerate and senile plaques accumulate (1). This squarely places the synapse as a target in the early pathogenesis of AD. Because synapses exist in a dynamic microenvironment with nonneuronal cells, glial and endothelial cells have been implicated as targets and participants in the progression of $\mathrm{AD}(2,3)$. For example, $\mathrm{A} \beta$ treatment has been shown to alter the ability of astrocytes to ameliorate neuronal oxidative insults (4-6) and to have specific effects on mitochondrial respiration and cellular $\mathrm{Ca}^{2+}$ balance $(5,7)$. However, the $\mathrm{A} \beta$-mediated derailment of the functional and cellular interactions among the neurons, glia, and capillaries are insufficiently understood (8).

We are testing the hypothesis that one of the earliest Aß-mediated neurotoxic events may occur primarily in the mitochondria of perisynaptic glia, altering glial cell $\mathrm{Ca}^{2+}$ homeostasis $(5,9)$. In this scenario, $A ß$ subsequently induces neurodegeneration by predisposing nerve terminals to activityinduced damage following reduction of the supportive/protective capacity of the glia that sheath the nerve terminal, or perhaps by exposure to substances secreted from the microvascular endothelium. Most studies that have addressed mechanisms of $A \beta$-induced toxicity have used primary cell culture models or relatively complex model systems comprised of multiple cell types. We have taken a different approach to study the initiation and progression of the earliest events of Aß-induced neurotoxicity on intracellular and intercellular function. We have developed organotypic slice and pure nerve terminal or glial cell culture preparations of the rat posterior pituitary (PP), and are using this simplified model system in combination with cellular imaging methods employing $\mathrm{Ca}^{2+}$ sensitive or membrane potential-sensitive dyes that selectively localize to specific sub-cellular compartments. The PP preparation has the advantage in that it is comprised almost solely of brain nerve terminals (without axons or cell bodies), a network of a single type of astrocyte (pituicyte), and a rich microvasculature.

We used confocal methods to probe the morphological characteristics of the main players in this model system, and have begun to characterize functional aspects of the interaction between these components. The energy states of single or populations of individual mitochondria in nerve terminals or pituicytes were assessed by digital fluorescence imaging or high-throughput fluorescence plate reader and JC-1, a fluorescent ratiometric indicator of mitochondrial inner membrane potential. Similar results were obtained using the mitochondrial selective potentiometric dye TMRM. Following $24 \mathrm{hr}$ treatment with $25 \mu \mathrm{M}$ A325-35, isolated pituicytes exhibited on average $\sim 70 \%$ decrease in ratio values compared to vehicle- or reverse peptide-treated control values. The level of mitochondrial inhibition after $24 \mathrm{hr}$ treatment was comparable to that induced by treatment with the $20 \mu \mathrm{M}$ FCCP, a protonophore that uncouples mitochondria. Surprisingly, treatment of isolated nerve terminals with $\mathrm{A} \beta$ did not produce a significant decrease in ratio values compared to control. Likewise, activity induced changes in mitochondrial $\mathrm{Ca}^{2+}$ import, as measured by the mitochondria-selective $\mathrm{Ca}^{2+}$ dyes Rhod-2, $-\mathrm{FF}$ and $-5 \mathrm{~N}$, were decreased after $\mathrm{A} \beta$ treatment in pituicytes but not in nerve terminals. Consistent with the differential effects of $A ß$ on mitochondrial 
inner membrane potential and mitochondrial $\mathrm{Ca}^{2+}$ dynamics, $24 \mathrm{hr}$ Aß-treated pituicytes exhibited impaired cytosolic $\mathrm{Ca}^{2+}$ homeostasis as compared to control cells. Treated and control nerve terminals responded to $\mathrm{Ca}^{2+}$ challenges similarly. Using fluorescently tagged $\mathrm{A} \beta$ peptides, we demonstrated that differences between pituicytes and terminals in the uptake or trafficking of exogenous $A ß$ might in part underlie the observed sensitivity of pituicytes to $A ß$

1. Selkoe, D. J. (2002) Science 298(5594), 789-791

2. Grammas, P., Moore, P., and Weigel, P. H. (1999) Am J Pathol 154(2), 337-342

3. Paradisi, S., Sacchetti, B., Balduzzi, M., Gaudi, S., and Malchiodi-Albedi, F. (2004) Glia 46(3), 252-260

4. Nagele, R. G., D'Andrea, M. R., Lee, H., Venkataraman, V., and Wang, H. Y. (2003) Brain Res 971(2), 197-209

5. Abramov, A. Y., Canevari, L., and Duchen, M. R. (2003) J Neurosci 23(12), 5088-5095

6. Abramov, A. Y., Canevari, L., and Duchen, M. R. (2004) J Neurosci 24(2), 565-575

7. Pascale, A., and Etcheberrigaray, R. (1999) Pharmacol Res 39(2), 81-88

8. Lo, E. H., Broderick, J. P., and Moskowitz, M. A. (2004) Stroke 35(2), 354-356

9. Haughey, N. J., and Mattson, M. P. (2003) Neuromolecular Med 3(3), 173-180 